\title{
Relationship between the Membrane Inhibitor of Reactive Lysis and the Erythrocyte Phenotypes of Paroxysmal Nocturnal Hemoglobinuria
}

\author{
M. H. Holguin, ${ }^{\star}$ L. A. Wilcox, ${ }^{\star}$ N. J. Bernshaw, ${ }^{\star}$ W. F. Rosse, ${ }^{\star}$ and C. J. Parker ${ }^{\star}$ \\ *Department of Medicine, University of Utah School of Medicine, and the Veterans Administration Medical Center, Salt Lake City, \\ Utah 84148; and ${ }^{\ddagger}$ Department of Medicine, Duke University Medical Center, Durham, North Carolina 27710
}

\begin{abstract}
Susceptibility to hemolysis initiated by activated cobra venom factor (CoF) complexes is a characteristic that distinguishes the most complement-sensitive type III erythrocytes of paroxysmal nocturnal hemoglobinuria (PNH) from the intermediately sensitive type II and the normally sensitive type I cells. Recently we isolated a membrane constituent from normal erythrocytes that inhibits CoFBb-initiated hemolysis, and this protein was designated membrane inhibitor of reactive lysis (MIRL). To investigate the molecular basis of the variability in complement sensitivity among PNH erythrocytes, the surface expression of MIRL and decay accelerating factor (DAF) on the three phenotypes of PNH was quantified immunochemically. Both complement regulatory proteins were markedly deficient on the erythrocytes from a patient with predominately type III cells. The erythrocytes from patients with a majority of either type II or I cells were also significantly deficient in both MIRL and DAF. While cytofluorometric analysis confirmed the quantitative deficiencies, segregation of erythrocytes into discrete subpopulations that expressed either no MIRL or normal amounts of MIRL was not observed. The results of immunoprecipitation studies were consistent with quantitative, but not qualitative abnormalities of MIRL and DAF. Selective removal of the sensitive erythrocytes indicated that $\sim 20 \%$ of the normal amount of MIRL is sufficient to protect cells from CoF-initiated lysis. These studies suggest that relatively subtle quantitative differences in membrane complement regulatory proteins underlie the variability in complement sensitivity of PNH erythrocytes.
\end{abstract}

\section{Introduction}

Paroxysmal nocturnal hemoglobinuria $(\mathrm{PNH})^{1}$ is an acquired hemolytic anemia in which the erythrocytes are abnormally

Address correspondence to Dr. Charles J. Parker, Hematology/Oncology Section (111C), Veterans Administration Medical Center, 500 Foothill Drive, Salt Lake City, UT 84148. 1989.

Received for publication 17 March 1989 and in revised form 9 May

1. Abbreviations used in this paper: AET, 2-(aminoethyl)isothiuronium bromide; $\mathrm{CB}$, cell blank; $\mathrm{CBC}$, cell blank controlled for spontaneous hemolysis in the presence of serum; CLS, complement lysis sensitivity; CoF, cobra venom factor; $\mathrm{CoFBb}$, activated $\mathrm{CoF}$ complexes in which activated factor $\mathrm{B}(\mathrm{Bb})$ is bound to $\mathrm{CoF}$ forming a stable $\mathrm{C} 3 / \mathrm{C} 5$ convertase; $\mathrm{C} 8 \mathrm{bp}, \mathrm{C} 8$ binding protein; DAF, decay accelerating factor; GVB, Veronal-buffered saline containing $0.1 \%$ gelatin; $\mathrm{HRF}$, homologous restriction factor; MAC, membrane attack complex; MIRL, membrane inhibitor of reactive lysis; NHS, normal

J. Clin. Invest.

(c) The American Society for Clinical Investigation, Inc.

$0021-9738 / 89 / 11 / 1387 / 08 \quad \$ 2.00$

Volume 84, November 1989, 1387-1394 susceptible to complement-mediated hemolysis (1). The syndrome appears to arise as a result of a somatic mutation involving the pluripotential hematopoietic stem cell, since the leukocytes and platelets of PNH are also abnormal. A precise in vitro assay designed to quantify the complement sensitivity of the erythrocytes showed that the cells can be segregated into discrete subpopulations that differ in susceptibility to hemolysis mediated by the classical pathway. The following phenotypic classification of PNH erythrocytes is based on this assay: PNH I, normal or nearly normal in sensitivity; PNH II, intermediately sensitive; and PNH III, markedly sensitive.

Compelling evidence indicates that the greater hemolytic susceptibility of PNH erythrocytes is due to deficiencies of membrane constituents that regulate the functional activity of complement. Decay accelerating factor (DAF) is a $70-\mathrm{kD}$ glycoprotein that limits the formation and stability of the C3 convertases of the classical and alternative pathways $(2,3)$. PNH III erythrocytes are completely deficient in DAF (4-6). In some cases PNH II erythrocytes appear to be missing DAF entirely, while in others the deficiency may be either partial (4) or qualitative (7). Quantitatively abnormal DAF expression has also been reported for PNH I erythrocytes (6). PNH III erythrocytes are also deficient in $\mathrm{C} 8$ binding protein (C8bp) homologous restriction factor (HRF; 8, 9), a 65-kD protein that inhibits the cytolytic activity of the membrane attack complex (MAC; 10, 11). Analysis of expression of HRF/C8bp by PNH I and PNH II erythrocytes has not been reported.

Susceptibility to complement-mediated hemolysis initiated by cobra venom factor ( $\mathrm{CoF}$ ) also distinguishes PNH III erythrocytes from PNH I and II cells (12). In this process (sometimes called reactive or bystander lysis), complement is activated by an alternative pathway $\mathrm{C} 3 / \mathrm{C} 5$ convertase (CoFBb) that is formed when factor $\mathrm{B}$ that has bound to $\mathrm{CoF}$ (a functional analogue of human $C 3 b ; 13$ ) is enzymatically activated by factor D. PNH III erythrocytes are readily lysed when incubated with $\mathrm{CoFBb}$ and serum (the complement source) because the cells fail to restrict formation of the cytolytic MAC of complement (12). In contrast, PNH I, PNH II, and normal erythrocytes are resistant to this process because assembly of the MAC is inhibited. We have recently isolated an $18-\mathrm{kD}$ protein from normal erythrocytes (designated membrane inhibitor of reactive lysis [MIRL]) that protects PNH III erythrocytes from CoFBb-initiated hemolysis (14). By blocking the function of MIRL with a monospecific antibody, normal erythrocytes were made susceptible to reactive lysis, and immunoblotting experiments showed that PNH III erythrocytes were deficient in MIRL. Those studies indicated that the susceptibility of PNH III erythrocytes to CoFBb-initiated hemo-

human serum; PNH, paroxysmal nocturnal hemoglobinuria; RIP, radioimmunoprecipitation; TBS, Tris-buffered saline; VBS, Veronalbuffered saline. 
lysis is causally related to a deficiency of MIRL. Based on their resistance to reactive lysis, we hypothesized that MIRL is present on PNH I and II erythrocytes. Accordingly, studies aimed at defining the relationship between MIRL and the erythrocyte phenotypes of PNH have been undertaken.

\section{Methods}

Erythrocytes. Whole blood from volunteer donors and patients with PNH was obtained by venopuncture and stored in Alsever's solution at $4^{\circ} \mathrm{C}$. Normal erythrocytes were treated with 2-(aminoethyl)isothiuronium bromide (AET; 15) or trypsin (16) using previously described methods.

Buffers. The following buffers were used: Veronal-buffered saline (VBS) containing $5 \mathrm{mM}$ sodium barbital (Sigma Chemical Co., St. Louis, MO), $\mathrm{pH} 7.5$, and $145 \mathrm{mM} \mathrm{NaCl}$ (VBS); VBS containing $0.1 \%$ gelatin (GVB); GVB containing $15 \mathrm{mM}$ sodium salt of EDTA (GVBEDTA); $5 \mathrm{mM}$ sodium phosphate, $\mathrm{pH} 8.0$, containing $1 \mathrm{mM}$ PMSF (Sigma Chemical Co.) and $1 \mathrm{mM}$ EDTA (5,8 P-PE); $10 \mathrm{mM}$ sodium

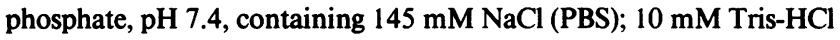
(Sigma Chemical Co.), pH 7.4, containing $145 \mathrm{mM} \mathrm{NaCl}$, (TBS); TBS containing $0.1 \%$ gelatin, $1.0 \%$ NP-40 (Sigma Chemical Co.), and $1 \mathrm{mM}$ PMSF (radioimmunoprecipitation [RIP] buffer). Normal human serum (NHS) chelated with EDTA was prepared by mixing EDTA (10 $\mathrm{mM}$ final concentration) and incubating the mixture for $5 \mathrm{~min}$ at $37^{\circ} \mathrm{C}$. The NHS-EDTA sample was kept at $4^{\circ} \mathrm{C}$ before use.

Activated CoF complexes. CoF from Naja naja naja (Miami Serpenterium, Salt Lake City, UT; 17), factor B (18), and factor D (18) were isolated according to previously described methods. Activated $\mathrm{CoF}$ complexes (CoFBb) were prepared by incubating $100 \mu \mathrm{l}$ of $\mathrm{CoF}$ $(200 \mu \mathrm{g} / \mathrm{ml})$ with $100 \mu \mathrm{l}$ of factor $\mathrm{B}(1 \mathrm{mg} / \mathrm{ml}), 50 \mu \mathrm{l}$ of factor $\mathrm{D}$, and 50 $\mu l$ of GVB containing $5 \mathrm{mM} \mathrm{MgCl}{ }_{2}$ at $37^{\circ} \mathrm{C}$. After 30 min excess $\mathrm{Mg}^{2+}$ was chelated by adding $40 \mu \mathrm{l}$ of $0.1 \mathrm{M}$ EDTA, pH 7.4, and continuing the incubation for $5 \mathrm{~min}$. The complexes were stored at $-70^{\circ} \mathrm{C}$ and diluted 1:5 with GVB-EDTA before use.

Antibodies. Antiserum to MIRL was prepared by injecting a New Zealand white rabbit subcutaneously with Freund's complete adjuvant containing $5 \mu \mathrm{g}$ of purified MIRL. Two subsequent injections with $5 \mu \mathrm{g}$ of MIRL in Freund's incomplete adjuvant were given at 2-wk intervals. 2 wk after the last injection whole blood was obtained by venopuncture and the serum was isolated. Nonimmune serum was obtained from New Zealand white rabbits before immunization. Rabbit anti-DAF antiserum was prepared as previously described (19). The $F\left(a b^{\prime}\right)_{2}$ fragment of affinity-purified donkey anti-rabbit IgG that had been labeled with ${ }^{125}$ I was purchased from Amersham Corp. (Arlington Heights, IL). Affinity-purified goat anti-rabbit IgG conjugated with FITC was purchased from Sigma Chemical Co.

Determination of protein concentration. The concentrations of $\mathrm{CoF}$ and factor B were determined spectophotometrically (17). All other protein concentrations were determined with the BCA protein assay (Pierce Chemical Co., Rockford, IL) using BSA as the standard.

Radiolabeling. Normal and PNH erythrocytes were surface-labeled with ${ }^{125}$ I as NaI (Amersham Corp.) using IODO-GEN (Pierce Chemical Co.; 16).

SDS-PAGE and autoradiography. Analysis of proteins by SDSPAGE (16) was performed using linear gradient gels of 6-15\%. Molecular weights were estimated (20) by reference to standards purchased from Bio-Rad Laboratories (Richmond, CA). Autoradiographs (16) were prepared by exposing dried gels to X-Omat AR film (Eastman Kodak Co., Rochester, NY) at $-70^{\circ} \mathrm{C}$.

Complement lysis sensitivity (CLS) assay. PNH erythrocytes were characterized according to their sensitivity to antibody-initiated complement-mediated hemolysis as described by Rosse and Dacie $(21,22)$. The effects of inhibition of MIRL function on the complement sensitivity of normal erythrocytes was assessed by treating cells with antiMIRL antiserum. Erythrocytes were washed in GVB-EDTA and resuspended to $5 \times 10^{8} / \mathrm{ml}$. Aliquots of the cells were incubated at $37^{\circ} \mathrm{C}$ with GVB-EDTA containing either a saturating concentration of anti-MIRL antiserum or an equal volume of nonimmune rabbit serum. After 30 min the cells were washed and used in the CLS assay.

Susceptibility of normal and PNH erythrocytes to complement-mediated hemolysis initiated by CoFBb. Normal and PNH erythrocytes were washed three times in GVB-EDTA and resuspended to $5 \times 10^{8}$ / $\mathrm{ml}$. Seven twofold serial dilutions of NHS-EDTA were prepared using GVB-EDTA as the diluent. 100- $\mu$ l aliquots of the serum dilutions were incubated at $37^{\circ} \mathrm{C}$ with $50 \mu \mathrm{l}$ of cells and $50 \mu \mathrm{l}$ of CoFBb. To control for spontaneous hemolysis, a cell blank (CB) was prepared by combining $50 \mu \mathrm{l}$ of cells and $150 \mu \mathrm{l}$ of GVB-EDTA. The reagent blank that controlled for spontaneous hemolysis in the presence of serum (CBC) contained erythrocytes and the appropriate serum dilution, but GVBEDTA was substituted for the CoFBb complexes. Hemolysis of $100 \%$ of the cells was achieved by substituting $0.04 \%$ ammonium hydroxide for the other reagents. After $30 \mathrm{~min} 3 \mathrm{ml}$ of GVB-EDTA was added and the samples were centrifuged at $1,000 \mathrm{~g}$ for $3 \mathrm{~min}$. Hemolysis was quantified by measuring free hemoglobin in the supernates (aliquots of $200 \mu \mathrm{l}$ were pipetted into the wells of microtiter plates and the $A_{410}$ of the samples was measured with a microplate reader ([MR 700; Dynatech Laboratories, Inc., Alexandria VA]). The following formula was used to calculate the percent hemolysis:

$\frac{A_{410} \text { of sample }-A_{410} \text { of } C B C}{A_{410} \text { of } 100 \%-A_{410} \text { of } C B} \times 100$.

Comparison of the sensitivity of PNH III and anti-MIRL-treated normal erythrocytes to CoFBb-initiated hemolysis. Normal and PNH erythrocytes (from patient R.O.) were washed in GVB-EDTA and resuspended to $5 \times 10^{8} / \mathrm{ml}$. A 1-ml aliquot of normal erythrocytes was incubated at $37^{\circ} \mathrm{C}$ with $1 \mathrm{ml}$ of GVB-EDTA containing a saturating concentration of anti-MIRL antiserum. After $30 \mathrm{~min}$ the cells were washed in GVB-EDTA and resuspended to $1 \mathrm{ml}$. Next, 50- $\mu$ l aliquots of PNH or anti-MIRL-treated cells were incubated at $37^{\circ} \mathrm{C}$ with $50 \mu \mathrm{l}$ of $\mathrm{CoFBb}$ and $100 \mu \mathrm{l}$ of GVB-EDTA containing incremental concentrations of NHS-EDTA. Controls (the CB, CBC, and $100 \%$ lysis) were prepared as described above. After $30 \mathrm{~min} 3 \mathrm{ml}$ of GVB-EDTA was added to each sample and hemolysis quantified as described above.

Quantification of the amount of anti-MIRL required to induce sensitivity of erythrocytes to CoFBb-initiated hemolysis. Normal and PNH erythrocytes were washed in GVB-EDTA and resuspended to $5 \times 10^{8}$ \% ml. $200-\mu \mathrm{l}$ aliquots of cells were incubated at $37^{\circ} \mathrm{C}$ with $200 \mu \mathrm{l}$ of GVB-EDTA containing incremental concentrations of anti-MIRL antiserum. As controls, cells were also incubated with GVB-EDTA alone and with GVB-EDTA containing nonimmune rabbit serum. After $30 \mathrm{~min}$ the cells were washed with GVB-EDTA and resuspended to $200 \mu \mathrm{l} .50-\mu \mathrm{l}$ aliquots of cells were incubated at $37^{\circ} \mathrm{C}$ with $100 \mu \mathrm{l}$ of NHS-EDTA and $50 \mu \mathrm{l}$ of CoFBb. After $30 \mathrm{~min}$ hemolysis was quantified as described above.

Binding of anti-MIRL and anti-DAF to normal and PNH erythrocytes. Normal and PNH erythrocytes were washed three times in GVB-EDTA and resuspended to $5 \times 10^{8} / \mathrm{ml}$. $50-\mu \mathrm{l}$ aliquots of cells were incubated at $37^{\circ} \mathrm{C}$ with $50 \mu \mathrm{l}$ of GVB-EDTA that contained a saturating concentration of anti-MIRL or anti-DAF antiserum. Saturating concentrations were determined in preliminary experiments by generating binding isotherms for the two antibodies. To control for nonspecific binding, an equal amount of nonimmune rabbit serum was substituted for the specific antisera. After $30 \mathrm{~min}$ the samples were washed with GVB-EDTA and resuspended to $100 \mu \mathrm{l}$. An aliquot of 100 $\mu \mathrm{l}$ of GVB-EDTA that contained a saturating amount of ${ }^{125}$ I-donkey anti-rabbit IgG was added to each sample, and the reaction mixtures were incubated for $30 \mathrm{~min}$ at $37^{\circ} \mathrm{C}$. Next, two $75-\mu \mathrm{l}$ aliquots from each sample were pipetted onto a $200-\mu 1$ layer of a combination of phthalate esters in $400-\mu l$ polyethylene microfuge tubes. After centrifugation at $8,000 \mathrm{~g}$ in a microfuge (model 12; Beckman Instruments Inc., Fullerton, CA), the tips of the microfuge tubes containing the cells were cut off, and the radioactivity of the pellet was quantified in a gamma counter. 
Quantitation of binding of anti-MIRL to AET- and trypsin-treated erythrocytes was performed using the same methods.

Binding of anti-DAF and anti-MIRL to PNH erythrocytes after CoFBb-initiated hemolysis. Normal and PNH erythrocytes were washed in GVB-EDTA and resuspended to $5 \times 10^{8} / \mathrm{ml}$. A 1-ml aliquot of the PNH cells was incubated at $37^{\circ} \mathrm{C}$ with $1 \mathrm{ml}$ of $\mathrm{CoFBb}, 500 \mu \mathrm{l}$ of NHS-EDTA, and $1.5 \mathrm{ml}$ of GVB-EDTA. After $30 \mathrm{~min}$ the unlysed cells were washed in GVB-EDTA and resuspended to $6 \times 10^{7} / \mathrm{ml}$. The control cells (normal and PNH erythrocytes that had not been exposed to $\mathrm{CoFBb}$ and serum) were also diluted to $6 \times 10^{7} / \mathrm{ml}$. Next, binding of anti-MIRL and anti-DAF was quantified as described above.

RIP of normal and PNH erythrocyte membrane proteins with anti$M I R L$ and anti-DAF. Normal and PNH erythrocytes that had been surface-labeled with ${ }^{125}$ I were washed and resuspended to $5 \times 10^{8} / \mathrm{ml}$ in PBS. 2-ml aliquots were centrifuged at $1,000 \mathrm{~g}$ for $3 \mathrm{~min}$ and the supernate was aspirated. Hemoglobin-free erythrocyte ghosts were prepared by washing the cells in hypotonic buffer (5,8 P-PE; 16). The radiolabeled ghosts were resuspended to $1 \mathrm{ml}$ in 5,8 P-PE, and a $250-\mu \mathrm{l}$ aliquot was pipetted into a separate microfuge tube and kept at $4^{\circ} \mathrm{C}$ before analysis by SDS-PAGE and autoradiography. A 750- $\mu$ l aliquot of RIP buffer was added to the remaining ghosts, the suspension was incubated for $10 \mathrm{~min}$ at $37^{\circ} \mathrm{C}$ and $20 \mathrm{~min}$ on ice, and then centrifuged for $5 \mathrm{~min}$ at $12,000 \mathrm{~g}$. Next, the supernate was recovered and incubated overnight at $4^{\circ} \mathrm{C}$ with $500 \mu \mathrm{l}$ RIP buffer containing $10 \%$ (vol/vol) protein A-Sepharose CL-4B (Sigma Chemical Co.). The suspensions were centrifuged at $12,000 \mathrm{~g}$ for $2 \mathrm{~min}$, the supernate was recovered, and the volume was adjusted to $2 \mathrm{ml}$ with RIP buffer. Next, $500-\mu \mathrm{l}$ aliquots were pipetted into $1.5-\mathrm{ml}$ microfuge tubes and $10 \mu \mathrm{l}$ of antiMIRL, anti-DAF, or nonimmune rabbit serum were added. After incubation for $30 \mathrm{~min}$ at $37^{\circ} \mathrm{C}, 100 \mu \mathrm{l}$ of RIP buffer containing $10 \%$ protein A-Sepharose was added, and the samples were incubated at room temperature. After $30 \mathrm{~min}$ the samples were centrifuged at $12,000 \mathrm{~g}$ for $2 \mathrm{~min}$ and the supernate was aspirated. The protein A-Sepharose beads were washed three times with RIP buffer. After the final wash the supernate was aspirated as completely as possible without disturbing the pellet, $50 \mu \mathrm{l}$ of reduced sample buffer (16) was added, and the tubes were placed in a boiling water bath for $5 \mathrm{~min}$. Next, the samples were centrifuged for $5 \mathrm{~min}$ at $12,000 \mathrm{~g}$ and the supernates were recovered and subjected to analysis by SDS-PAGE and autoradiography.

Cytofluorographic analysis of MIRL and DAF on normal and PNH erythrocytes. Normal and PNH erythrocytes were washed three times in GVB-EDTA and resuspended to $2 \times 10^{7} / \mathrm{ml}$. 50- $\mu 1$ aliquots of cells were incubated at $37^{\circ} \mathrm{C}$ with $50 \mu \mathrm{l}$ of GVB-EDTA containing a saturating concentration of anti-MIRL, anti-DAF, or an equivalent amount of nonimmune rabbit serum. After $30 \mathrm{~min}$ the cells were washed in GVB-EDTA and resuspended to $50 \mu \mathrm{l}$. Next, $50 \mu \mathrm{l}$ of a 1:40 dilution of FITC-conjugated goat anti-rabbit IgG was added and the reaction mixtures were incubated at $37^{\circ} \mathrm{C}$. After $30 \mathrm{~min}$ the cells were washed in GVB-EDTA and resuspended to $1 \mathrm{ml}$. The samples were analyzed with a cytofluorograph II (Ortho Diagnostic Systems, Inc., Raritan, $\mathrm{NJ})$. A minimum of 5,000 cells were counted.

\section{Results}

Characterization of the complement sensitivity of PNH erythrocytes. Two methods were used to test the susceptibility of the erythrocytes from the three patients with PNH to complement-mediated hemolysis (Table I). The standard method for classifying PNH erythrocytes is the CLS. In this assay erythrocytes are sensitized with an optimal amount of anti-IgM (isolated from a patient with chronic cold agglutinin disease), and dilutions of NHS serve as the complement source. The relative sensitivity of erythrocytes is defined by the amount of serum required to induce hemolysis of $50 \%$ of a particular population of cells. Accordingly, the erythrocytes of PNH have been clas-
Table I. Characterization of the Complement Sensitivity of the PNH Erythrocytes

\begin{tabular}{lcccc}
\hline Patient & PNH I* & PNH II & PNH III* & $\begin{array}{c}\text { Maximum hemolysis } \\
\text { induced by CoFBb }\end{array}$ \\
\hline & & \multicolumn{3}{c}{} \\
R.O. & 0 & 0 & $>95$ & 84 \\
B.A. & 29 & 64 & 7 & 9 \\
O.B & 79 & 0 & 21 & 21 \\
\hline
\end{tabular}

* Determined in the CLS assay.

${ }^{\ddagger}$ Derived from the results illustrated in Fig. 2.

sified into the following three groups: PNH I cells are equivalent or (nearly equivalent) in sensitivity to normal erythrocytes; PNH II cells require $25-33 \%$ as much serum and are therefore considered to be intermediate in sensitivity; and PNH III cells are markedly sensitive, requiring 4-7\% as much serum for an equal degree of lysis compared with normal erythrocytes. The results of the CLS assay for the erythrocytes used in this study are shown in Table I.

To determine the effects of inhibition of MIRL function on susceptibility to hemolysis mediated by the classical pathway of complement, normal erythrocytes were incubated with either anti-MIRL antiserum or nonimmune rabbit serum. After washing, the cells were used in the CLS assay (Fig. 1). For comparison, the CLS curve for the erythrocytes of patient R.O. is shown. The anti-MIRL treatment increased the sensitivity of normal erythrocytes to that of PNH III cells. These results

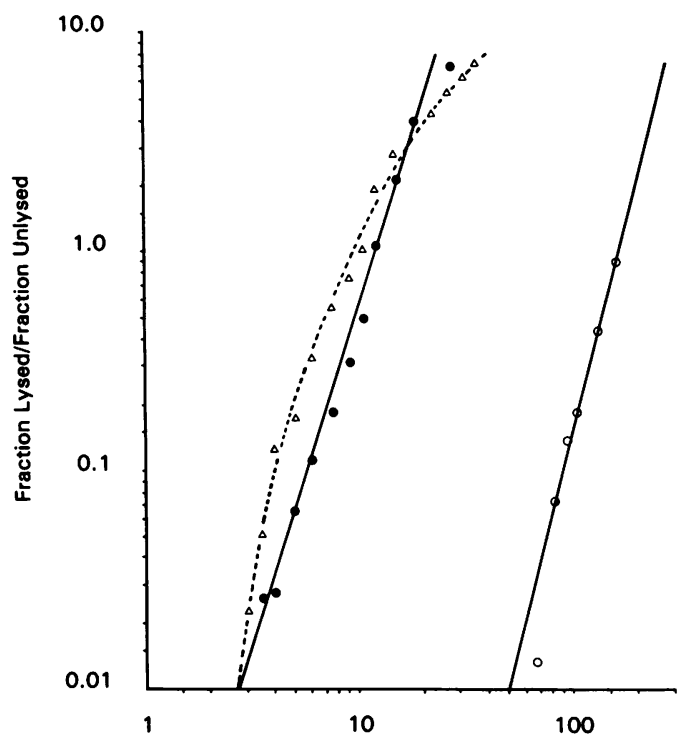

Equivalent Milliliters of Serum Diluted 1:270

Figure 1. Comparison of the susceptibility of normal, anti-MIRLtreated normal, and PNH III erythrocytes to hemolysis in the CLS assay. Normal erythrocytes were incubated with buffer containing either saturating amounts of anti-MIRL antiserum (•) or an equivalent amount of nonimmune rabbit serum (0). After washing, the cells were used in the CLS assay along with the erythrocytes from patient R.O. ( $\Delta$ ). By blocking MIRL function, normal erythrocytes were made as susceptible to antibody-initiated complement-mediated hemolysis as PNH III cells. 
indicate that MIRL is an important inhibitor of antibody-initiated hemolysis as well as of reactive lysis. In the CLS assay the IgM antibody is used in excess with the complement source being the limiting factor. Thus, direct activation of the classical pathway by anti-MIRL would not influence the result of the assay. We also observed that by allowing purified MIRL to incorporate into the cells (14), PNH III erythrocytes isolated by cell-affinity chromatography (6) can be made to manifest a CLS profile similar to that of PNH I cells (data not shown).

In the second assay used to assess the complement sensitivity of the PNH erythrocytes, $\mathrm{CoFBb}$ were used to initiate hemolysis (Fig. 2). The maximum susceptibility of the PNH erythrocytes to $\mathrm{CoFBb}$-initiated hemolysis was similar to the percentage of cells that were classified as type III in the CLS assay (Table I).

Previous studies have shown that MIRL protects PNH erythrocytes from $\mathrm{CoFBb}$-initiated hemolysis and that $\mathrm{PNH}$ III erythrocytes are deficient in MIRL (14). In addition, PNH III erythrocytes have been shown to be deficient in HRF/C8bp $(8,9)$. Based on its reported function, HRF/C8bp would be expected to offer some protection against $\mathrm{CoFBb}$-initiated hemolysis. To test this possibility, MIRL function was blocked by treating normal erythrocytes with optimal amounts of anti-MIRL antiserum. After washing, the susceptibility of the anti-MIRL-treated cells to $\mathrm{CoFBb}$-initiated hemolysis was compared with that of the PNH III cells. As illustrated in Fig. 3, the susceptibility of the anti-MIRL-treated cells was equivalent to that of the PNH III cells. Identical results were observed when purified anti-MIRL IgG was used instead of anti-MIRL antiserum, but isolated IgG from a nonimmune rabbit had no effect on the susceptibility of normal cells to CoFBb-initiated hemolysis (not shown). These experiments demonstrate that the susceptibility of PNH III cells to CoFBb-initiated hemolysis is due primarily to a deficiency of MIRL.

Comparison of surface expression of MIRL and DAF on $P N H$ erythrocytes by using a radioimmunobinding assay. Monospecific polyclonal antibodies were used to quantitate the amount of DAF and MIRL on the PNH erythrocytes (Fig. 4). The erythrocytes from all three patients demonstrated a

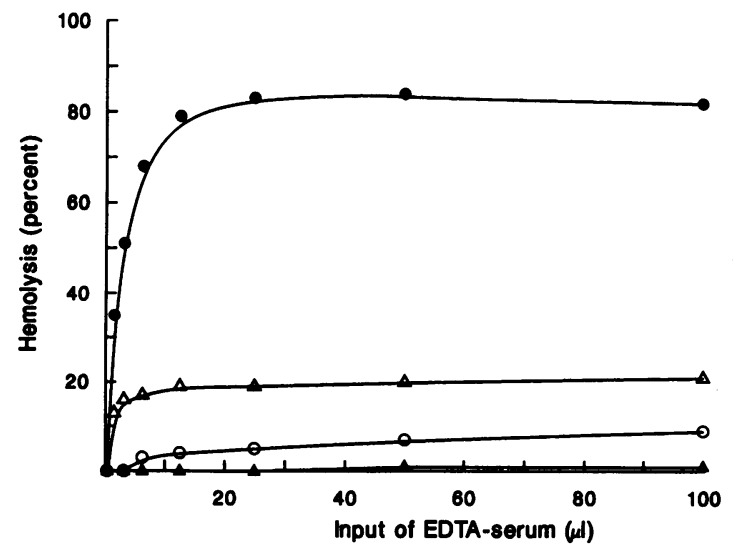

Figure 2. CoFBb-initiated hemolysis of PNH and normal erythrocytes. Normal ( $\Delta$ ) and PNH erythrocytes from patients B.A. (O), O.B. $(\Delta)$, and R.O. (๑) were incubated with $\mathrm{CoFBb}$ and incremental concentrations of EDTA-chelated serum. Hemolysis was subsequently quantified by measuring free hemoglobin. The data points represent the mean of triplicate determinations. Susceptibility to $\mathrm{CoFBb}$-initiated hemolysis is a characteristic of PNH III erythrocytes.

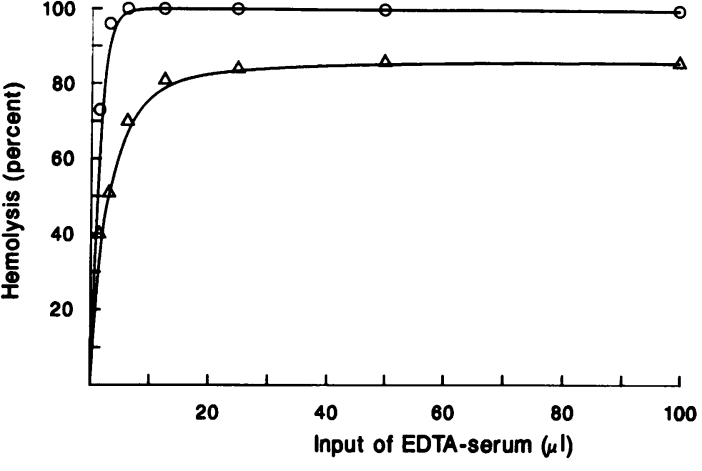

Figure 3. Comparison of PNH III and anti-MIRL-treated normal erythrocytes to $\mathrm{CoFBb}$-initiated hemolysis. Normal erythrocytes were incubated with a saturating amount of anti-MIRL antiserum. After washing, the antibody-treated cells $(0)$ and the erythrocytes from patient R.O. $(\Delta)$ were incubated with $\mathrm{CoFBb}$ and incremental concentrations of EDTA-chelated serum and hemolysis subsequently quantified. The data points represent the mean of triplicate determinations. By blocking MIRL function, normal erythrocytes are made as susceptible to CoFBb-initiated hemolysis as PNH III cells.

parallel deficiency of both regulatory proteins. Among volunteer donors, the number of molecules of DAF/erythrocyte $(\sim 2,000)$ was reported to be relatively uniform (6). Uniformity of expression also appears to be the case for MIRL, because by using the indirect binding assay the counts per minute for anti-MIRL-treated erythrocytes from 10 volunteer donors was $29,944 \pm 3,460$ (mean $\pm \mathrm{SD}, n=5$ for each subject, range of means $=25,666-36,662)$.

Normal erythrocytes treated with the sulfhydryl reagent, AET, are susceptible to CoFBb-initiated hemolysis (12), but trypsin-treated erythrocytes are not (Parker, C. J., unpublished observations). When AET-treated cells were assessed for surface expression of MIRL, no antibody binding was observed (Fig. 5). In contrast, trypsin treatment produced a modest reduction in binding of anti-MIRL. These results are consistent with the hypothesis that AET disrupts the structural/functional integrity of MIRL and thereby induces susceptibility to reactive lysis.

Cytofluorographic analysis of the surface expression of MIRL on PNH erythrocytes. To determine if MIRL expression among PNH I, II, and III erythrocytes is discrete and homogeneous, cells were analyzed using the FACS (Fig. 6). Consistent with the phenotypic profile of the patient's erythrocytes (Table I), the cells of patient R.O. demonstrated a marked deficiency of MIRL (Fig. $6 \mathrm{C}$ ). Analysis of the erythrocytes of patient B.A. (Fig. $6 \mathrm{~B}$ ), however, were inconsistent with the concept that PNH II cells express a normal amount of MIRL. Rather, except for a modest quantitative difference, the pattern of MIRL expression on B.A.'s erythrocytes was similar to that of patient R.O. The FACS analysis of the erythrocytes of patient O.B. was also more consistent with a continuum of MIRL expression than with division into discrete subpopulations with either normal or no MIRL expression (Fig. $6 D$ ).

Analysis by using RIP of MIRL and DAF on PNH erythrocytes. These studies were performed to determine if MIRL and DAF are qualitatively as well as quantitatively abnormal on PNH erythrocytes. The deficiencies of MIRL observed in the RIP studies (Fig. 7 B) paralleled those seen in the radioimmunobinding studies (Fig. 4), and no qualitative differences of 

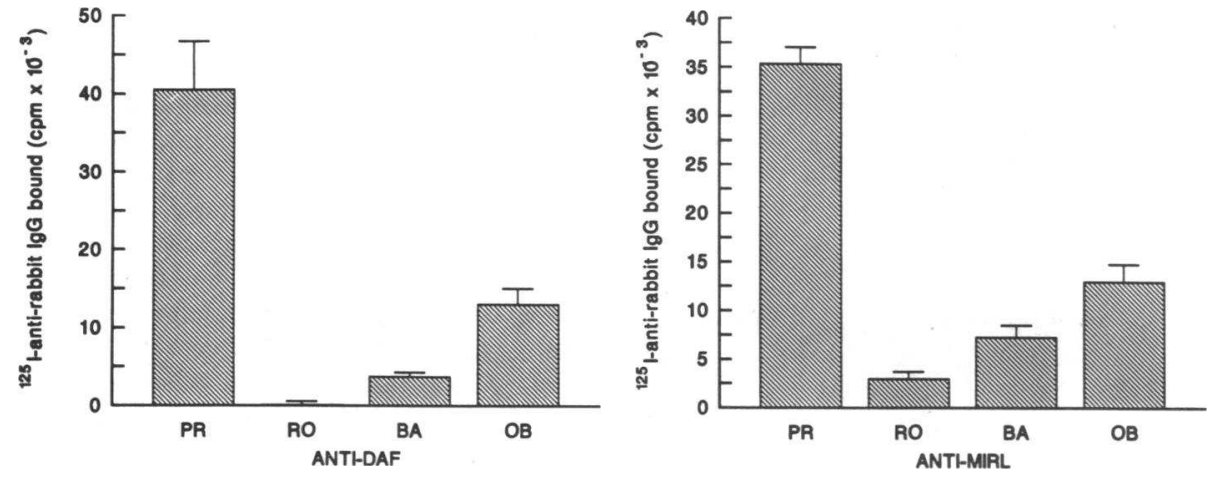

Figure 4. Quantitation of erythrocyte DAF and MIRL by using a radioimmunobinding assay. Normal $(P R)$ and PNH erythrocytes from patients R.O., B.A., and O.B. were incubated with either saturating amounts of antiDAF or anti-MIRL antiserum or with an equal amount of nonimmune rabbit serum (to control for nonspecific binding). Subsequently, binding of rabbit IgG was quantified by incubating the cells with a saturating amount of ${ }^{125} \mathrm{I}$ anti-rabbit IgG. The values (specific binding) depicted by the bars represent the mean $+\mathrm{SD}(n=5)$. The PNH erythrocytes demonstrate a parallel deficiency of DAF and MIRL.
MIRL were apparent. Similar results were obtained with antiDAF except that a diffuse band was observed in the molecular weight range $\leq 14 \mathrm{kD}$ in the lane containing the immunoprecipitate from patient R.O. (Fig. $7 \mathrm{~B}$, lane 3 ). The nature of this band is unknown, but it was not identified by anti-DAF in Western blot analysis (not shown).

Effects of anti-MIRL on the susceptibility of PNH erythrocytes to CoFBb-initiated hemolysis. The preceding studies have suggested that the difference in complement sensitivity among PNH I, II, and III cells is not due to the existence of discrete subpopulations of cells, one of which is completely deficient in a complement regulatory protein, while the other expresses a normal amount. This conclusion was directly challenged by pretreating the erythrocytes from a volunteer donor and from patients B.A. and O.B. with incremental concentrations of anti-MIRL. After washing, the cells were analyzed for susceptibility to $\mathrm{CoFBb}$-initiated hemolysis. The experimental conditions were designed so that the dose-response curve for the anti-MIRL-treated normal erythrocytes manifested a sigmoid appearance (Fig. 8). If a subpopulation of erythrocytes bearing a normal amount of MIRL existed

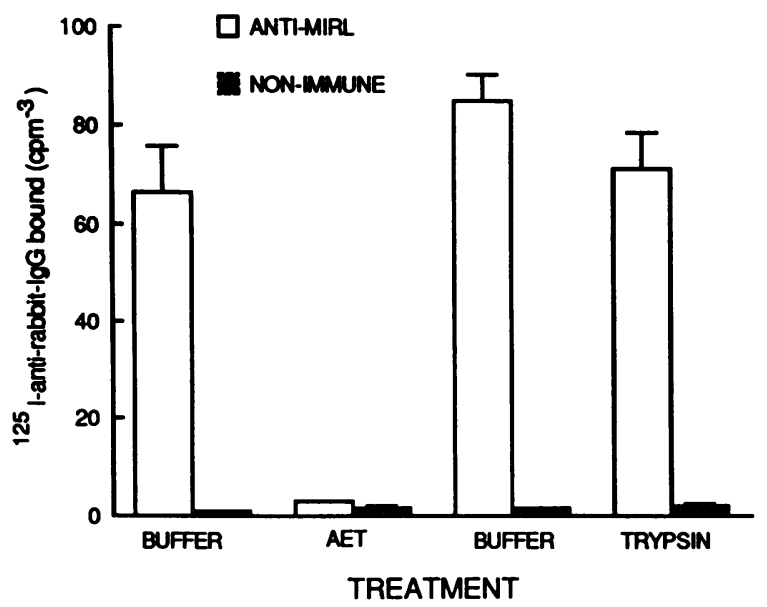

Figure 5. Binding of anti-MIRL to AET and trypsin-treated erythrocytes. The binding of anti-MIRL to normal erythrocytes treated with AET, trypsin, or buffer was assessed using the same methods as described in the legend to Fig. 4. The values depicted by the bars represent the mean $+\operatorname{SD}(n=5)$. Treatment of cells with AET completely abolished the binding of anti-MIRL, whereas trypsin treatment had little effect. among the PNH erythrocytes, that subpopulation should produce a dose-response curve that would parallel that of the anti-MIRL-treated normal erythrocytes. This, however, was not the case. These results suggest that the entire population of erythrocytes from patients B.A. and O.B. are deficient in MIRL, and that the erythrocytes that are hemolyzed in the absence of anti-MIRL treatment are simply more profoundly deficient.

Quantitation of MIRL on PNH erythrocytes after CoFBbinitiated hemolysis. To examine further the question of whether the erythrocytes that are insusceptible to hemolysis induced by CoFBb possess normal amounts of MIRL, DAF, or both, PNH cells were incubated with CoFBb and NHSEDTA. Next, the unlysed cells were recovered and the amount of MIRL and DAF on these insensitive cells was quantified using the radioimmunobinding assay. At the same time, the

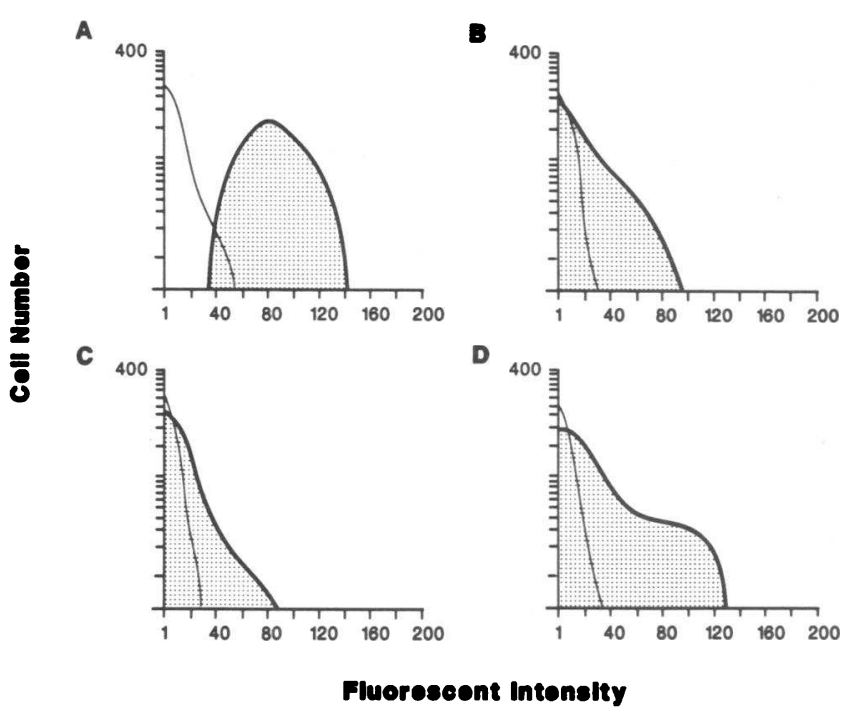

Figure 6. FACS analysis of anti-MIRL binding to normal and PNH erythrocytes. Normal $(A)$ and PNH erythrocytes from patients B.A. $(B)$, R.O. $(C)$, and O.B. $(D)$ were incubated with either a saturating amount of anti-MIRL antiserum (shaded area) or an equal amount of nonimmune rabbit serum (area under the curve inscribed by the thinner of the two lines). After washing, the cells were incubated with a saturating amount of FITC-conjugated anti-rabbit IgG. A minimum of 5,000 cells was analyzed for each subject. A bimodal cytofluorometric pattern was not observed for the erythrocytes of patients B.A. and O.B. 

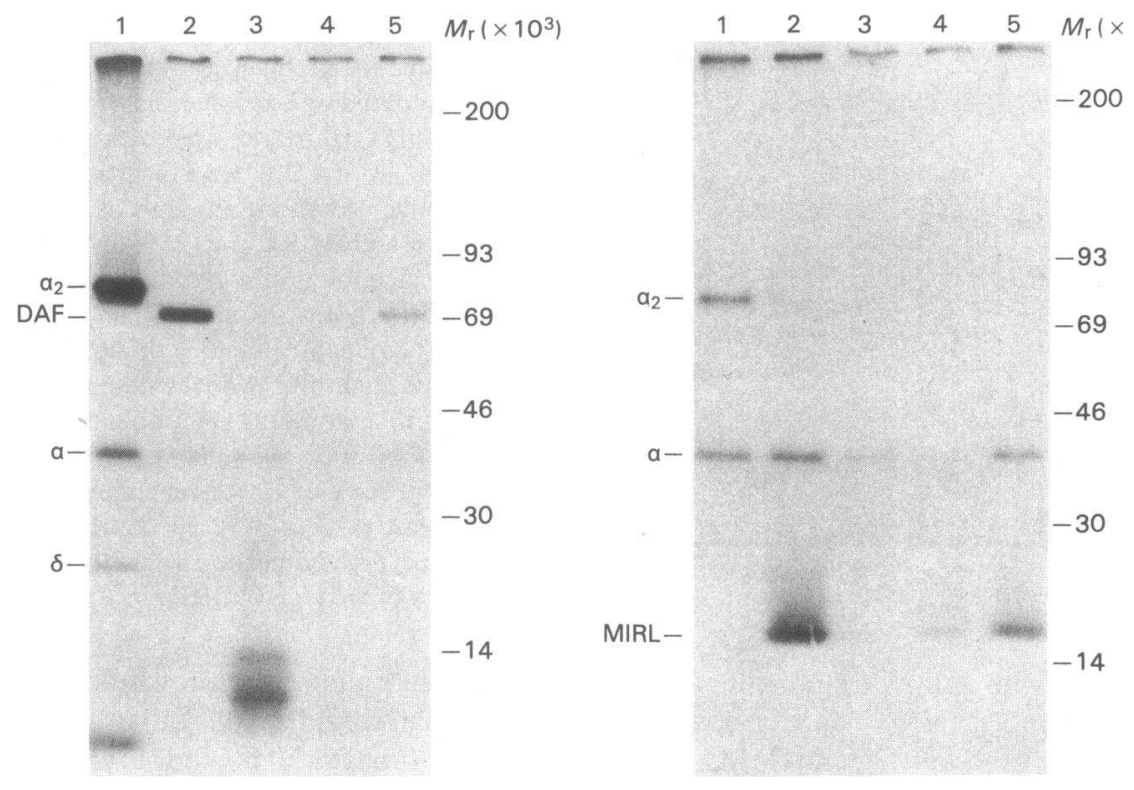

Figure 7. Analysis of DAF and MIRL on normal and PNH erythrocytes by using RIP. Hemoglobin-free ghosts of normal and PNH erythrocytes were prepared from cells that had been surface-labeled with ${ }^{125}$ I. Membrane proteins were solubilized and immunoprecipitated by using anti$\operatorname{DAF}(A)$ or anti-MIRL $(B)$. Lane 1 , radiolabeled ghosts from normal erythrocytes. The other lanes contain RIP from the following sources: lane 2, normal volunteer; lane 3, patient R.O.; lane 4, patient B.A.; lane 5, patient O.B. The Greek letters denote the positions of the glycophorin- $\alpha$ monomer and dimer and of the $\delta$ monomer. The positions of the molecular weight standards and of DAF and MIRL are also indicated. Parallel quantitative abnormalities of DAF and MIRL are observed for the patients with PNH.

amount of MIRL and DAF present on the erythrocytes before selective lysis of the sensitive cells was also determined. Among the PNH patients, only the erythrocytes from R.O. expressed a significantly greater amount of MIRL after selective removal of the CoFBb-sensitive cells (Fig. 9). This amount of MIRL, however, remained markedly less than normal. In agreement with the other studies reported herein, these results suggest that the degree of complement sensitivity of erythrocytes from patients with PNH is due to relatively subtle quantitative variations in the amount of regulatory proteins present on the erythrocyte membrane surface.

\section{Discussion}

The studies reported here suggest that PNH II erythrocytes are deficient in MIRL as well as DAF. Previously, either of two hypotheses seemed plausible as explanations for the molecular basis of the differences between the PNH II and III pheno-

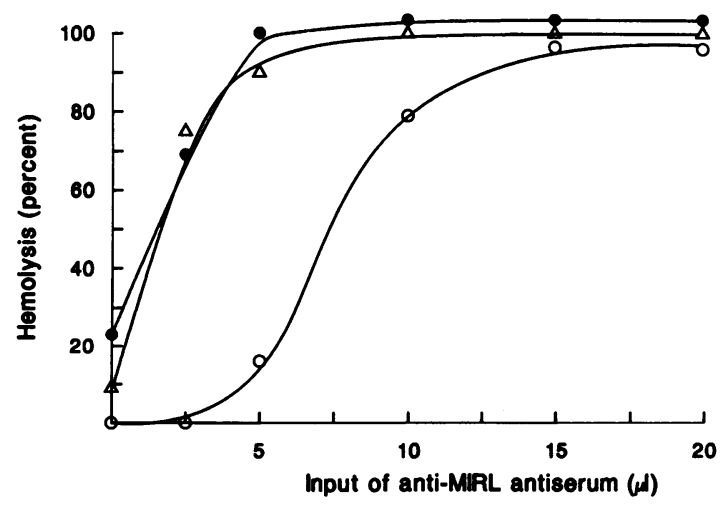

Figure 8. CoFBb-initiated hemolysis of normal and PNH erythrocytes treated with anti-MIRL. Normal (O) and PNH erythrocytes from patients B.A. $(\Delta)$ and O.B. $(\bullet)$ were incubated with incremental amounts of anti-MIRL. After washing, the cells were incubated with an optimal amount of CoFBb and EDTA-chelated serum, and hemolysis was subsequently quantified. The data points depict the mean of triplicate determinations. Greater amounts of anti-MIRL are required to block MIRL function on normal erythrocytes than on PNH erythrocytes. types. According to the first hypothesis, the two phenotypes have the same basic membrane defect, with the PNH III cells being more severely affected. In the second hypothesis, PNH III erythrocytes have two independent defects. One deficiency accounts for the intermediate complement sensitivity and the greater activation and binding of $\mathrm{C} 3 \mathrm{~b}$, and this defect is shared by PNH II cells. The absence of a second complement regulatory protein is responsible for the greater susceptibility of PNH III cells to CoFBb-initiated hemolysis, and this other factor is normal on PNH II erythrocytes. The combined deficiency of the two regulatory proteins accounts for the marked sensitivity of PNH III erythrocytes in the CLS assay.

Inasmuch as both DAF and MIRL are markedly deficient on the erythrocytes of patient B.A. (Fig. 4), only the first of the hypotheses stated above appears tenable. This patient has only 7\% type III cells (Table I and Fig. 2), yet the erythrocytes expressed $\sim 20 \%$ of the normal amount of MIRL. If the second hypothesis were correct, erythrocytes from a patient with 93\% CoFBb-insensitive cells (64\% type II and 29\% type I) would have essentially normal amounts of MIRL.

The results of the experiments shown in Fig. 9 suggest that $\sim 20 \%$ of the normal amount of MIRL inhibits CoFBb-initiated hemolysis because this amount was present on the insensitive cells of patient R.O. The erythrocytes that are classified as PNH II appear to have an amount of MIRL that is just above the threshold that provides protection against reactive lysis (Figs. 4 and 9). Combined with a parallel DAF deficiency, however, this same degree of MIRL deficiency apparently causes the cells to display an intermediate sensitivity in the CLS assay (the PNH II phenotype).

The fact that PNH I erythrocytes are nearly normal in susceptibility to hemolysis mediated by the classical pathway has suggested that they may be the progeny of the residuum of the normal clone, and that among patients the normal and abnormal cells coexist in various proportions (1). Analysis of MIRL and DAF associated with the erythrocytes of patient O.B., however, demonstrate that PNH I erythrocytes are abnormal. This patient has $80 \%$ type I cells, and yet quantitative binding studies show that the erythrocytes express 37 and $32 \%$ of the normal amount of MIRL and DAF, respectively (Fig. 4). 

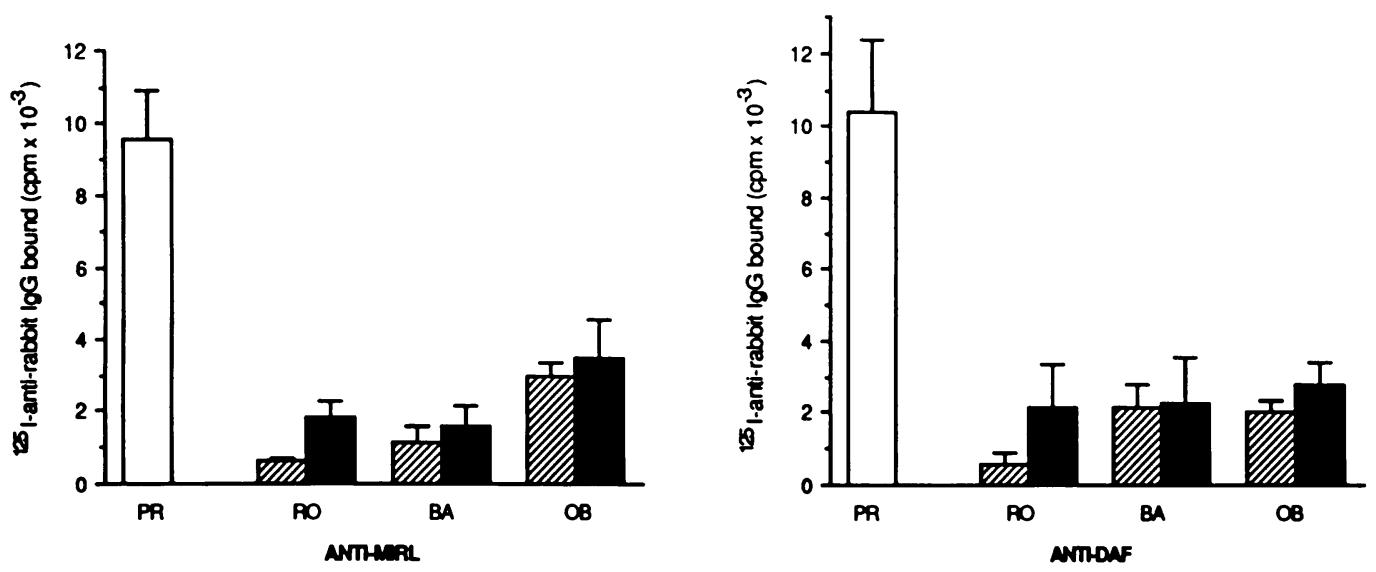

Figure 9. Quantitation of MIRL and DAF on PNH erythrocytes that are insusceptible to $\mathrm{CoFBb}$-initiated hemolysis. Erythrocytes from patients R.O., B.A., and O.B. were incubated with optimal amounts of $\mathrm{CoFBb}$ and NHS-EDTA, and the insensitive cells were subsequently recovered. Next, binding of anti-MIRL and anti-DAF to the unlysed population (closed bars) was compared with binding to the unfractionated population (cells that had not been incubated with CoFBb and NHS-EDTA; hatched bars) and to normal erythrocytes (open bars). The results depicted by the bars represent the mean $+\operatorname{SD}(n=5)$. The insensitive erythrocytes are deficient in MIRL and DAF.

Further, the cytofluorographic analysis of MIRL expression demonstrates that only $47 \%$ of the patient's erythrocytes have a fluorescence intensity that falls within the normal range, and that the cells do not display the bimodal pattern expected if two distinct populations exist (Fig. $6 \mathrm{D}$ ).

Two other lines of evidence indicate that PNH I erythrocytes are abnormal. First, the amount of antibody that was required to block MIRL function on the cells that were initially insusceptible to $\mathrm{CoFBb}$-initiated hemolysis was much less than that required for inhibition of MIRL function on normal erythrocytes (Fig. 8). Second, when the PNH III erythrocytes of patients O.B., R.O., and B.A. were selectively hemolyzed, the residual PNH I cells where observed to be deficient in MIRL and DAF (Fig. 9). Based on studies that compared expression of DAF and acetycholine esterase by PNH erythrocytes that were insusceptible to $\mathrm{CoFBb}$-initiated hemolysis, Medof and colleagues also concluded that PNH I cells are abnormal (6). Thus it appears that by the time the PNH syndrome becomes evident clinically, normal erythrocytes have been replaced almost entirely by the progeny of the abnormal clone. The mechanisms that account for the heterogeneity of expression of the various membrane proteins among patients remain speculative.

Despite the fact that they are significantly deficient in complement regulatory proteins, PNH I erythrocytes demonstrate a nearly normal susceptibility to antibody-initiated hemolysis. These observations suggest that on normal erythrocytes MIRL and DAF are present in a relative excess. Apparently the degree of deficiency of the inhibitory constituents must be profound before in vitro and in vivo evidence of disordered regulation of complement is manifested.

Normal erythrocytes treated with the sulfhydryl reagent AET have been used as models for investigating the pathophysiological basis of PNH (1). In the CLS assay the chemically modified cells display a susceptibility similar to that of PNH III erythrocytes (15). Further, like PNH III cells AETtreated erythrocytes are susceptible to $\mathrm{CoFBb}$-initiated hemolysis, and after incubation with CoFBb and NHS-EDTA they bind relative large amounts of the trimolecular $\mathrm{C} 5 \mathrm{~b}-7$ complex (12). In immunoblotting studies anti-MIRL does not bind to MIRL if the SDS-PAGE is performed under reducing condition (Parker, C. J., unpublished observations), and normal erythrocytes that have been treated with AET no longer bind
anti-MIRL (Fig. 5). Taken together, these observations suggest that AET destroys the structural and functional integrity of MIRL by disrupting intrachain disulfide bonds. While AET may affect other erythrocyte constituents (23), abolition of MIRL function appears to account for most of the disordered regulation of complement, because the behavior of normal erythrocytes in which MIRL function has been blocked by antibody is analogous to that of the chemically modified cells (Figs. 1 and 3).

Previous studies have suggested that AET does not disrupt the structural or functional integrity of DAF (23). Normal DAF function may account for the observation that AETtreated erythrocytes do not bind as much C3 as PNH II and III erythrocytes after activation of the classical pathway (12). As evidenced by the results illustrated in Fig. 1, however, control of the classical pathway C3 convertase by DAF appears to offer relatively little protection against complement-mediated hemolysis if MIRL is not functional.

The relationship between the expression of HRF/C8bp and the erythrocyte phenotypes of PNH has not been analyzed in this report. Our studies suggest, however, that HRF/C8bp has a limited capacity to control complement-mediated hemolysis initiated either by the classical pathway activation (Fig. 1) or by $\mathrm{CoFBb}$ (Fig. 3) because anti-MIRL-treated normal erythrocytes are as sensitive as PNH III erythrocytes (cells that are presumably missing $\mathrm{HRF} / \mathrm{C} 8 \mathrm{bp}$ ). In the studies reported here, $\mathrm{CoFBb}$ and serum have been used to induce reactive lysis. Sugita et al. (24) have isolated a complement regulatory protein that appears to be identical to MIRL. In those studies, the $18-\mathrm{kD}$ protein was shown to block reactive lysis induced by isolated $\mathrm{C} 5 \mathrm{~b} 6, \mathrm{C} 7, \mathrm{C} 8$, and $\mathrm{C} 9$, but an inhibitor with the reported properties of $\mathrm{HRF} / \mathrm{C} 8 \mathrm{bp}$ could not be identified. We have made similar observations.

The molecular basis of PNH remains enigmatic. To date, all of the proteins that are deficient in PNH have been shown to share the common biochemical feature of being anchored to the cell membrane by a glycosyl-phosphatidylinositol moiety (25-31). This observation has led to the hypothesis that PNH is the manifestation of an abnormality in the complex systems of either synthesis, attachment, or degradation of phosphatidylinositols. Accordingly, studies to determine if MIRL is anchored by a phosphatidylinositol-glycan are being pursued $(32,33)$. 


\section{Acknowledgments}

The authors wish to thank Dr. Jarmila Janatova and Dr. John Bohnsack for critically reviewing the manuscript. Dr. John P. Atkinson (Washington University School of Medicine, St. Louis, MO) kindly provided the anti-DAF antiserum. The excellent technical assistance of Sharon Hall (Duke University Medical Center, Durham, NC) is gratefully acknowledged.

This work was supported by the Research Service of the Veterans Administration, by Public Health Service grants 5-RO1-DK-35830-05 from the National Institute of Diabetes and Digestive and Kidney Diseases (awarded to Dr. Parker), and 5-R37-DK-31379-07 from the National Heart Lung and Blood Institute (awarded to Dr. Rosse).

\section{References}

1. Rosse, W. F., and C. J. Parker. 1985. Paroxysmal nocturnal haemoglobinuria. Clin. Haematol. 14:105-125.

2. Nicholson-Weller, A., J. Burge, D. T. Fearon, P. F. Weller, and F. Austen. 1982. Isolation of a human erythrocyte glycoprotein with decay accelerating activity for $\mathrm{C} 3$ convertases of the complement system. J. Immunol. 129:184-189.

3. Medof, E. M., T. Kinoshita, and V. Nussenzweig. 1984. Inhibition of complement activation on the surface of cells after incorporation of decay-accelerating factor (DAF) into their membranes. J. Exp. Med. 160:1558-1578.

4. Nicholson-Weller, A., J. P. March, S. I. Rosenfeld, and K. F. Austen. 1983. Affected erythrocytes of patients with paroxysmal nocturnal hemoglobinuria are deficient in the complement regulatory protein decay-accelerating factor. Proc. Natl. Acad. Sci. USA. 80:5066-5070.

5. Pangburn, M. K., R. D. Schrieber, and H. J. Müller-Eberhard. 1983. Deficiency of an erythrocyte membrane protein with complement regulatory activity in paroxysmal nocturnal hemoglobinuria. Proc. Natl. Acad. Sci. USA. 80:5430-5434.

6. Medof, M. E., A. Gottlieb, T. Kinoshita, S. Hall, R. Silber, V. Nussenzweig, and W. F. Rosse. 1987. Relationship between decay accelerating factor deficiency, acetylcholinesterase activity, and defective terminal complement pathway restriction in paroxysmal nocturnal hemoglobinuria erythrocytes. J. Clin. Invest. 80:165-174.

7. Kinoshita T., S. I. Rosenfeld, and V. Nussenzweig. 1987. A high m. w. form of decay-accelerating factor (DAF-2) exhibits size abnormalities in paroxysmal nocturnal hemoglobinuria erythrocytes. J. Immunol. 138:2994-2998.

8. Zalman, L. S., L. M. Wood, M. M. Frank, and H. J. MüllerEberhard. 1987. Deficiency of the homologous restriction factor in paroxysmal nocturnal hemoglobinuria. J. Exp. Med. 165:572-577.

9. Hänsch, G. M., S. Schönermark, and D. Roelcke. 1987. Paroxysmal nocturnal hemoglobinuria type III. Lack of an erythrocyte membrane protein restricting the lysis by C5b-9. J. Clin. Invest. $80: 7-12$

10. Schönermark, S., E. W. Rauterberg, M. L. Shin, S. Loke, D. Roelcke, and G. M. Hänsch. 1986. Homologous species restriction in lysis of human erythrocytes: a membrane-derived protein with C8binding capacity functions as an inhibitor. J. Immunol. 136:17721776.

11. Zalman, L. S., L. M. Wood, and H. J. Müller-Eberhard. 1986. Isolation of a human erythrocyte membrane protein capable of inhibiting expression of homologous complement transmembrane channels. Proc. Natl. Acad. Sci. USA. 82:7711-7715.

12. Parker, C. J., T. Wiedmer, P. J. Sims, and W. F. Rosse. 1985. Characterization of the complement sensitivity of paroxysmal nocturnal hemoglobinuria erythrocytes. J. Clin. Invest. 75:2074-2084.

13. Vogel, C.-W., C. A. Smith, and H. J. Müller-Eberhard. 1984. Cobra venom factor: structural homology with the third component of human complement. J. Immunol. 133:3235-3241.

14. Holguin, M. H., L. R. Fredrick, N. J. Bernshaw, L. A. Wilcox, and C. J. Parker. 1989. Isolation and characterization of a membrane protein from normal human erythrocytes that inhibits reactive lysis of the erythrocytes of paroxysmal nocturnal hemoglobinuria. J. Clin. Invest. 84:7-17.

15. Sirchia, G., and J. V. Dacie. 1967. Immune lysis of AET-treated normal red cells (PNH-like cells). Nature (Lond.). 215:747-748.

16. Parker, C. J., C. M. Soldato, and W. F. Rosse. 1984. Abnormality of glycophorin- $\alpha$ on paroxysmal nocturnal hemoglobinuria erythrocytes. J. Clin. Invest. 73:1130-1143.

17. Parker, C. J., V. F. White, and R. J. Falk. 1986. Site-specific activation of the alternative pathway of complement: synthesis of a hybrid molecule consisting of antibody and cobra venom factor. Complement. 3:223-235.

18. Parker, C. J., P. J. Baker, and W. F. Rosse. 1982. Increased enzymatic activity of the alternative convertase when bound to the erythrocytes of paroxysmal nocturnal hemoglobinuria. J. Clin. Invest. 69:337-346.

19. Seya, T., T. Farries, M. Nickells, and J. P. Atkinson. 1987. Additional forms of human decay-accelerating factor (DAF). J. Immunol. 139:1260-1267.

20. Weber, K., and M. Osborn 1969 . The reliability of molecular weight determinations by dodecyl-sulfate gel electrophoresis. J. Biol. Chem. 224:4406-4412.

21. Rosse, W. F., and J. V. Dacie. 1966. Immune lysis of normal human and paroxysmal nocturnal hemoglobinuria (PNH) red blood cells. I. The sensitivity of PNH red cells to lysis by complement and specific antibody. J. Clin. Invest. 45:736-748.

22. Rosse, W. F. 1973. Variations in the red cells in paroxysmal nocturnal haemoglobinuria. Br. J. Haematol. 24:327-342.

23. Parker, C. J., J. P. Atkinson, O. L. Stone, and N. J. Bernshaw. 1987. Treatment of erythrocytes with 2-aminoethylisothiouronium bromide abolishes the functional activity of the membrane receptor for complement C3b (CR1). Blood. 70:1 12a. (Abstr.)

24. Sugita, Y., Y. Nakaho, and M. Tomita. 1988. Isolation from human erythrocytes of a new membrane protein which inhibits the formation of complement transmembrane channels. J. Biochem. (Tokyo). 104:633-637.

25. Davitz, M. A., M. G. Low, and V. Nussenzweig. 1986. Release of decay-accelerating factor (DAF) from the cell membrane by phosphatidylinositol-specific phospholipase C (PIPLC). Selective modification of a complement regulatory protein. J. Exp. Med. 163:1150-1161.

26. Medof, M. E., E. I. Walter, W. L. Roberts, R. Haas, and T. L. Rosenberry. 1986. Decay accelerating factor of complement is anchored to cells by a C-terminal glycolipid. Biochemistry. 25:67406747.

27. Hänsch, G. M., P. F. Weller, and A. Nicholson-Weller. 1988. Release of $\mathrm{C} 8$ binding protein $(\mathrm{C} 8 \mathrm{bp})$ from the cell membrane by phosphatidylinositol-specific phospholipase C. Blood. 72:1089-1092.

28. Selvaraj, P., M. L. Dustin, R. Silber, M. G. Low, and T. A. Springer. 1987. Deficiency of lymphocyte function-associated antigen 3 (LFA-3) in paroxysmal nocturnal hemoglobinuria. J. Exp. Med. 166:1011-1025.

29. Burroughs, S. F., D. V. Devine, G. Browne, and M. E. Kaplan. 1988. The population of paroxysmal nocturnal hemoglobinuria neutrophils deficient in decay-accelerating factor is also deficient in alkaline phosphatase. Blood. 71:1086-1089.

30. Haziot, A., S. Chen, E. Ferrero, M. G. Low, R. Silber, and S. M. Goyert. 1988. The monocyte differentiation antigen, CD 14, is anchored to the cell membrane by a phosphatidylinositol linkage. $J$. Immunol. 141:547-552.

31. Selvaraj, P., W. F. Rosse, R. Silber, and T. A. Springer. 1988. The major Fc receptor in blood has a phosphatidylinositol anchor and is deficient in paroxysmal nocturnal haemoglobinuria. Nature (Lond.). 333:565-567.

32. Low, M. G., and A. R. Saltiel. 1988. Structural and functional roles of glycosyl-phosphatidylinositol in membranes. Science (Wash. DC). 239:268-275.

33. Ferguson, M. A. J., and A. F. Williams. 1988. Cell-surface anchoring of proteins via glycosyl-phosphatidylinositol structures. Annu. Rev. Biochem. 57:285-320. 